\title{
Les syllabes arrachées de Franc Ducros
}

\section{Margherita Orsino}

\section{(2) OpenEdition}

\section{Journals}

\section{Édition électronique}

URL : https://journals.openedition.org/studifrancesi/25901

DOI : 10.4000/studifrancesi.25901

ISSN : 2427-5856

\section{Éditeur}

Rosenberg \& Sellier

\section{Édition imprimée}

Date de publication : 1 avril 2007

Pagination : 117-127

ISSN : 0039-2944

\section{Référence électronique}

Margherita Orsino, «Les syllabes arrachées de Franc Ducros », Studi Francesi [En ligne], 151 (LI | I) | 2007, mis en ligne le 30 novembre 2015, consulté le 23 novembre 2021. URL : http://

journals.openedition.org/studifrancesi/25901; DOI : https://doi.org/10.4000/studifrancesi.25901

\section{(c) (i) (9)}

Studi Francesi è distribuita con Licenza Creative Commons Attribuzione - Non commerciale - Non opere derivate 4.0 Internazionale. 


\title{
Les syllabes arrachées de Franc Ducros
}

\author{
La poésie serait ce qui ne s'écrit pas, \\ mais que l'écrit révèle'.
}

La poésie de Franc Ducros appartient à cette lignée issue de Baudelaire, Rimbaud et Mallarmé, qui se poursuit, à travers le $\mathrm{Xx}^{\mathrm{e}}$ siècle avec les œuvres de Pierre Reverdy ${ }^{2} e t$ d'André Du Bouchet $^{3}$. Dans ses essais sur ces poètes, ainsi que dans son œuvre poétique 4 , Franc Ducros mène la recherche d'une poésie comme acte fondateur de la langue dans le rapport au réel. C'est une poésie qui n'a de cesse de s'interroger sur elle-même, tout aussi loin du lyrisme esthétisant que du jeu formel ou conceptuel. La poésie de Franc Ducros est, comme déjà celle d'André Du Bouchet, une poésie de l'expérience: expérience en tant que rapport au monde, en tant que rencontre avec l'obstacle qui nous fait buter et nous surprend, expérience de la parole comme acte primordial et fondateur surgissant de cette rencontre. Impact parfois brusque, voire douloureux, d'où naît une poésie qui creuse: «les inépuisables effets de la rencontre qui, nous déchirant, nous ouvre à toute connaissance... $\gg^{5}$. Ainsi, tout en la nommant, la poésie ne décrit pas la réalité mais se constitue en tant que rapport à elle, mouvement de et vers elle. Elle fait apparaître par la nomination un rapport qui est celui de soi - de l'être - au monde. D'où aussi une première difficulté d'étudier une poésie «dégagée du récit» qui depuis longtemps, depuis Baudelaire, «ne se laisse pas aussi volontiers réduire aux appareils critiques d'aujourd'hui. Elle leur résiste. Serait-ce de ne plus leur offrir la "prise" du récit qu'elle a congédié?»". Dans l'impossibilité de raconter, d'expliciter le mystère, chaque poème trace une sorte d'idéogramme: «signe qui est lui-même une liaison complexe entre des éléments, une articulation entre les éléments» ${ }^{7}$ donnant non seulement à voir mais à vivre le rapport au monde; d'où son aspect essentiel, sans ornements, foudroyant, qui entre tout de suite dans la matière de ce rapport, par des incipit tel que «mais», «comme», «où», etc.; d'où aussi son tracé parfois éclaté et intermittent, plastique, propre à ce langage poétique qui se présente comme une matière façonnable dont la syllabe serait la plus petite partie - la syllabe et non le signe, puisque c'est d'abord une poésie «dite» ${ }^{8}$. Encore

(1) F. Ducros, «Parole débordée». Lire André Du Bouchet dans Le Poétique et le Réel, Paris, Méridiens Klincksieck, 1987, p. 140.

(2) Pierre Reverdy (1889-1960) est, avec Max Jacob et Guillaume Apollinaire, l'un des fondateurs de la poésie moderne qu'il illustre dans sa revue «Nord-Sud». Si elle est d'abord étiquetée comme «cubiste», puis source d'inspiration pour les surréalistes, sa poésie et la réflexion poétique constante qui l'accompagne dépassent le cadre historique des avant-gardes et influencent encore aujourd'hui les poètes contemporains.

(3) André Du Bouchet (1924-2001), fondateur avec Bonnefoy, des Forêts, Dupin, de la revue «L'Ephémère» (1966-1973), proche de Reverdy et Celan, est l'une des voix les plus significatives de la poésie française contemporaine.
(4) Franc Ducros a mené parallèlement écriture critique et poétique (voir la note bio-bibliographique ci-contre). Dans ses études sur Baudelaire, Mallarmé, Reverdy, Du Bouchet entre autres, il retrace cette lignée dont il se réclame.

(5) F. Ducros, Poésie, figures traversées, SaintMaximin, Théétète, 1995, p.86.

(6) F. Ducros, Ibid. p. 45

(7) Entretien avec Alain Veinstein, Surpris par la nuit, France-Culture, 9. II. 04.

(8) «Avec Baudelaire - précise Franc Ducros - la langue française devient une langue de vocables, non plus de termes mais une langue et une voix qui dit». Ibid. 
faut-il l'extraire, l'arracher. Car, si elle se forme dans l'instant, elle peut également demander des années à se former. Aussi ce livre, dans son aspect suivi et instantané, n'a-t-il pourtant demandé pas moins de quinze ans de travail; son titre - une reprise des derniers mots du livre - nous indique le cheminement à la fois ardu et surprenant de la parole poétique.

Les syllabes arrachées se composent alors sur la feuille, figurant l'énergie dont elles sont issues, toujours sonores, vibrantes. Pas de majuscules, pas de ponctuation inutile: la dispositio impose la syntaxe, plus que des signes conventionnels pourraient le faire. Les poèmes se déploient noir sur blanc, mais un grand blanc contenant très peu de noir, où ils se détachent, parfois aériens et dispersés, parfois plus compacts au centre de la feuille, d'après une véritable mise en espace. Les blancs ne sont pas seulement des pauses, ce sont aussi des blancs de lumière qui peuvent éclairer des vers ou mots isolés; c'est aussi l'air où passe la parole comme un souffle, intervalles de la voix poétique, ou bien le vide auquel elle est parfois suspendue comme sur cet abîme d'où elle vient de surgir à l'instant. Le poème est vivant; il se dresse ou descend, parfois serpente telle la fracture située au centre du livre, suggérant ainsi une structure à deux temps, riche en contrastes et clair-obscur', ondulatoire comme la respiration, qui se laisse visualiser comme rupture ou ouverture, au creux ou au sommet de ce mouvement énergétique sinusoïdal:

\section{fracture}

comme à travers

l'air tord les branches

le travail obscur des racines

tu

resteras cette fracture

qui fait la terre soudain blanche

la nuit venue ${ }^{10}$.

Poésie en mouvement, donc, et poèmes comme passages - des profondeurs à la terre et à l'air, du noir à la couleur, du silence à la parole - dans un espace-temps qui, s'il fut d'abord celui de la sensation, est devenu celui de la traversée poétique. Poésie «à travers» - un des mots clés de ce livre - qui nous invite à franchir des seuils s'ouvrant sur l'inconnu. C'est ainsi «de seuil en seuil», pour citer Paul Celan ${ }^{11}$, un autre poète cher à Ducros, qu'il faut aborder ce livre conçu comme un tout et non comme un recueil ou un canzoniere, mais une composition en cinq mouvements et un final. Six moments d'un parcours à vitesse variable, dont les titres annoncent déjà les étapes et les rythmes.

(9) Compte tenu de l'importance de la mise en page, nous avons essayé de la respecter le plus possible lors des citations.

(10) F. Ducros, Surgies syllabes arrachées, Nîmes,
Théétète, 2003, p. 50.

(11) P. Celan, De seuil en seuil [1955], dans Choix de poèmes réunis par l'auteur, Paris, Gallimard, 1998. 


\section{Surgies...}

Regardons de plus près les titres des sectionset leur contenu:

1. du noir cela [un poème en sept mouvements]

2. les yeux, la terre [trois poèmes de longueur équilibrée, en plusieurs parties]

3. s'ouvrant [quatre «chapitres» intitulés: rouge - fracture - l'arbre - ce point ]

4. entre le feu et le soleil [trois poèmes en plusieurs parties]

5. l'oubli, l'éclat [quatre «chapitres» intitulés: Delphes - les yeux enfouis - taureaux - neige du 21 avril

6. chaude la terre s'éteignant [un unique poème de clôture]

On peut déjà entrevoir un cheminement de l'origine - le noir - à la sensation qui est avant tout visuelle et tactile - les yeux, la terre - jusqu'à la lumière et l'éclat, finissant par s'éteindre à nouveau.

Que l'origine soit genèse de la parole, reste gravé dans le poème liminaire, en sept parties, qui commence:

mais longtemps dans les plis

obscurs

$$
\text { - comme }
$$

sur des colonnes vaporeuses d'ombre

les nuages brillent dressés,

invisible est poussière profonde -

$$
\begin{aligned}
& \text { et la terre } \\
& \text { profonde - }
\end{aligned}
$$

en

sphères éclatant se dresse

la parole

nue $^{12}$.

Nue comme à l'origine, la parole est issue d'un espace-temps sans mémoire et sans histoire; essentielle, elle n'est pas encore revêtue d'autres significations culturelles, personnelles ou historiques. Elle est la première voix, «inouïe» au sens premier du mot:

(12) F. Ducros, Surgies syllabes arrachées, p. 7.

(13) F. Ducros, Parole sans attache, dans Poésie, figures traversées, p. 126. Il s'agit d'une étude critique sur la dernière poésie d'André Du Bouchet.

(14) «Sarà uno di quei tanti effetti di metamorfosi che ci fanno pensare che la parola è fatta di vocali e di consonanti, di sillabe, a un modo cioè del tutto diverso dagli oggetti che evoca e che possono essere oggetti lontani nello spazio? Lontani, tramontati nel tempo. Perfino appartenenti a epoche e a terre scomparse immemorabilmente. I E questo, l'unico dono di magia, il sommo potere di metamorfosi che abbia l'uomo? e il dono per cui la parola ci riconduce, nella sua oscura origine e nella sua oscura portata, al mistero, lasciandolo tuttavia inconoscibile, e come essa fosse sorta, si diceva, per opporsi in un certo senso, al mistero.» G. UngaretTI, Ragioni di una poesia, in Vita d'un uomo. Tutte le poesie, Milano, Mondadori (Oscar Grandi Classici), 2002, p. LXX. Ungaretti est l'un des poètes italiens qui ont beaucoup compté pour Franc Ducros, en particulier pour la question du rythme. Italianiste de formation, Ducros cherche à donner à la langue française un rythme, des accents, qu'il retrouve dans la poésie italienne et de façon particulière chez Ungaretti. Le poète français qui, comme ce dernier, a travaillé la langue par les syllabes est Mallarmé, qu'Ungaretti a d'ailleurs traduit. 
Dénudée la parole sans attache, c'est-à-dire sans antécédents, peut se faire, aux oreilles de plusieurs, voire de chacun, inaudible. Or il s'agit d'entendre. Non d'y réentendre ce qui fut. Voire ce qu'elle fut, quand - dans la chaleur encore vacante, non encore révolue - les points d'appui en monde ne s'étaient pas encore tous dérobés. Cela, depuis, et pour cela, est devenu audible: les points d'appui, apparus dans la durée du temps, ont rendu audible ce qui d'abord ne le fut pas. Corrélativement, ont obscurci ce qui, depuis, s'est élancé sans, désormais, les points d'appuis d'alors. Il se peut qu'aux lecteurs, alourdis de mémoire que nous risquons d'être, alors incapables d'entendre, à l'œuvre dans cette parole, l'oubli, les plus récentes émergences de cette parole, au sens strict inoü̈e $[\ldots]^{13}$.

L'avènement de la parole est alors perçu comme un accident, clinamen qui survient dans une durée et un espace indéterminés («longtemps dans les plis obscurs»); cette «déviation» est figurée par la conjonction adversative «mais» qui commence le poème en le reliant ainsi, tout en s'y opposant, à une condition prénatale impossible à décrire et même à imaginer comme forme. Ainsi la naissance de la parole est aussi naissance du temps (et contretemps); mieux, de la mesure du temps, du rythme, celui d'un cœur qui battrait pour la toute première fois.

Le noir figure l'abîme, le rien ou absence, gouffre sans mémoire d'où surgit la parole. C'est l'origine obscure, le «mystère» de la parole ungarettienne ${ }^{14}$, dépourvu cependant de toute idée de transcendance. Mais l'espace aussi, dans lequel la parole est lancée, est également abîme: «la parole qui fut, vers | le vide lancée | [...] dans | la respiration du jour I sinueuse s'abîme». Pénétrant le vide, la verbalisation du monde est avant tout une déchirure: «comme I du noir cela I évanouit la parole, se I déchirant le bleu | comble».

Ensuite, la parole devient mot, à savoir langue, donc logos:

mots

dans la main comme du cuir, comme l'odeur

de cheval dans le cuir d'une selle: ils

fuient et n'oublient pas. mais sur, rapide

ou lent, n'importe leur retour le mot, l'odeur

se changent. comme

moi comme un $\operatorname{mot}^{15}$.

C'est toute l'histoire des mots, toujours en devenir, mais gardant un souvenir de ce qu'ils ont été, comme une trace, une odeur, image chère à Ducros qui avait déjà intitulé son livre sur Dante, L'odeur de la panthère ${ }^{16}$ : «parole lancée en avant d'ellemême, à la poursuite de la langue, "panthère" toujours future dont, l'inventant, nous ne percevrons, loin devant nous que l'odeur». La langue poétique est une langue à inventer: «qui invente au présent la langue future» tout en laissant entendre la «langue mère dont elle est issue»; c'est pourquoi «la parole présente fait la langue toujours future en lançant en avant d'elle-même ce qui, vers elle, monte du passé de la langue $\gg^{17}$. Ainsi, comme chez Du Bouchet, et en amont encore, chez Reverdy, la

(15) F. Ducros, Surgies syllabes arrachées, p. 13.

(16) Ibid, L'odeur de la panthère, Saint-Maximin, Théétète, 1997. L'image est tirée du De vulgari eloquentia, (I,XVI) et placée en exergue de l'essai, p. 5:«panteram [...] redolentem ubique et necubi apparentem»; «panthère [...] odorant en tout lieu, en nul n'apparaissant».

(17) Ibid. p. 12. On retrouve une image équivalente dans les «stries» de Paul Celan: «stries, traînée dans l'œil:| que sois gardé I un signe porté par l'obscur, | animé.» P. CELAN, op. cit., p. 133. 
poésie reste toujours en-deçà de la réalité, constamment au bord d'un gouffre, d'un vide. Acte tragique, donc, celui de la création, celui: «de ne proférer qu'à la limite du possible, sur l'extrême bord» ${ }^{18}$.

Moi comme un mot: c'est l'être qui est lui aussi en transmutation dans l'acte d'inventer, être insaisissable, toujours à venir. C'est donc seulement à la conclusion de cette première section, que la première personne fait son apparition, annonçant ainsi la suivante, les yeux la terre, où s'amorce la relation entre le réel, la parole et le moi. Analogie qui laisse déjà entrevoir la dimension fusionnelle, d'appartenance. Le sujet n'est cependant pas encore exprimé en tant que «je», à savoir conscience, mais en tant que pure entité perceptive, en tant qu'yeux, voix, écoute, pied etc., réceptrice des toutes premières impressions vitales, figurées ici comme une pulsation chthonienne:

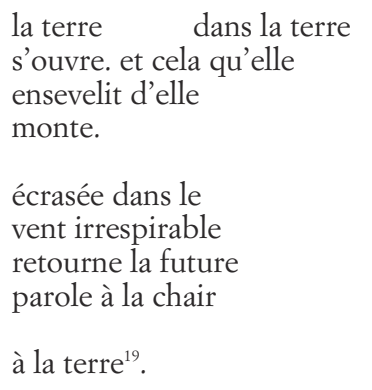

Le spasme vital est aussi un retournementde la matière - et du souffle - sur ellemême, dans la tentative d'une traversée ${ }^{20}$, une compénétration. Le corps (les yeux, la chair) et la terre (les éléments) finissent par se fondre:

l'écorce

ouverte respirant: les yeux

la terre dans

le sang se mêlent, s'excluent ${ }^{21}$.

Aussi l'expérience du réel est-elle une traversée; traversée de seuils invisibles entre les éléments:

tra-

versés par l'air des pays traversés les yeux

racines d'amandier ${ }^{22}$.

Le «je» prononcé une seule fois dans le premier mouvement - et très rarement dans toute cette poésie dépourvue d'un quelconque biographisme -, participe au

(18) Dans une étude sur Reverdy, Ducros le définit comme: «Poète tautologique: de ne proférer qu'à la limite du possible, sur l'extrême bord qui, basculant, révèle alors, un intant, au-dessous, en avant des choses nommées, "l'autre côté" - le "fond" dont elles sont issues, qui les donne et les emporte, les accueille aussi, et en elles tranparaît $[. ..] \gg$, Poésie, figures traversées, p. 86.

(19) F. Ducros, Surgies syllabas arrachées, p. 18.

(20) «Retourné, le sol fait surgir le fond, abîme. Faille extatique, le retournement est ce par quoi la terre s'ouvrant, le monde se donne en avènement d'événement. L'acte poétique s'articule à ce retournement: par lui le monde peut se déployer. La parole alors se donnant, porte en elle la marque du défaut d'où elle provient et que, pour exister et venir vers nous, elle a, se retournant depuis le fond, franchie. Figure du rapport au fond - émergence du rythme à même la parole. Qui retourne aussitôt au fond». F. Ducros, Poésie, figures traversées, p. 21.

(21) F. Ducros, Surgies syllabes arrachées, p. 19.

(22) Ibid., p. 22. 
bouleversement perpétuel, tout en restant cependant un élément, non nécessaire, de ce qui serait de toute manière arrivé: «[...] cela | a lieu. que | j’y sois cela | travail de terre a lieu. même | oublié. même $\mid$ moi mort ${ }^{23}$. Si le mystère lui échappe, c'est qu'il en fait partie: c'est qu'il est lui-même en jeu, tel que l'écrit Reverdy:

La vie entière est en jeu

Constamment

Nous passons à côté du vide élégamment sans tomber

Mais parfois quelque chose en nous fait tout trembler ${ }^{24}$.

Condition que Ducros définit: «connaissance d'un mystère redoutable, toujours proche, inéluctable et hors de portée, innommable mais - condition de tout nom et de toute chose, qui les porte toutes et tous, les traverse aussi et les compose ensemble, conférant à chacun et à leurs "rapports", la netteté et l'éclat de ce qui se dresse sur le sans-fond.... ${ }^{25}$. Même hors d'atteinte, le réel existe. Nous sommes ici aux antipodes du pari montalien ${ }^{26}$ : «cela a lieu», nul doute là-dessus, et ce, indépendamment de «moi». De même, la poésie, comme la réalité, vit par elle même; ni mémoire, ni «bistoire d'un homme», elle est objet palpitant qui déploie son rythme, son souffle.

\section{2. «Poèmes respirés»}

Que l'on remarque, d'après ces premiers exemples, les traits spécifiques de cette poésie: un tracé plastique où la disposition et le rythme, la scansion, font la poésie. Une poésie en torsion - par les nombreuses inversions - et en tension: entre des seuils, membranes invisibles entre le corps et le monde, entre le souffle et l'air, entre la voix et l'espace. Mais encore: un rythme vivant qui ouvre et ferme (la terre) ou qui expire et inspire (la parole). Enfin, une dimension vocale, faite d'harmonieuse alternance de voix et de silences, de déploiements et cassures, mais aussi d'une complexe trame syllabique, d'assonances et consonances, qui serpente elle aussi verticalement suivant cette versification «respirée».

Tout comme le mouvement «de» et «vers», tout comme le souffle, les poèmes présentent de nombreuses structures en miroir, en écho:

comme les yeux s'enfoncent dans la terre froide

que dresse la parole

l'air

d'où

venue l'herbe s'irise d'air

les yeux

de terre ${ }^{27}$.

Ils accomplissent ainsi l'impossible jonction de deux réalités: les yeux, la terre - l'herbe, l'air. Jointure qui est au cœur de l'image poétique, articulation cachée qui

(23) Ibid., p. 24.

(24) P. Reverdy, Galeries in Sources du vent, Main-d'œuvre, Paris, Mercure de France, 1949, pp. 125-127.
(25) F. Ducros, Poésie, figures traversées, pp. 84-85.

(26) E. Montale, Forse un mattino andando in un'aria di vetro..., dans Tutte le poesie, Milano, Mondadori, 1999, p. 42. 
peut aussi se penser «comme retournement de l'un dans l'autre, ou de l'un vers l'autre. Et se formuler selon la mesure du langage - en figures de chiasme, d'oxymoron, d'aporie, d'instant extatique... $\gg^{28}$. Ainsi, les images de traversée, ou de mêlée, sont multipliées jusqu'à la mise en abîme, jusqu'au tremblement et au vertige:

homme et nuage, contemplés, tournoient soir et matin, le bleu le gris se mêlent vides.

comme qui quand

tombe en rêve l'eau

monte verticale,

au silence. alors dressé le monde tout le bruit jusqu'

reste

enfoui ${ }^{29}$.

La verticalité de la plupart de ces poèmes semble suggérer la chute et l'ascension vertigineuse, entre deux abîmes en miroir, soulignées par la fréquence des verbes tel que: «sourdre», «se dresser», «se lever», «s'envoler», etc., qui côtoient «s'engouffrer», «s'abîmer», «enfouir», etc. En même temps, elle casse le phrasé, isolant les mots, voire les syllabes, en fragments, d'après un rythme qui n'est pas seulement celui de la perception ou de la pensée, mais celui de la voix poétique. Tel est l'enjeu de la poésie, celui de «convertir le vertige du gouffre en rythme se déployant $»^{30}$ :

\author{
comblés \\ de nuit les yeux, à \\ la nuit \\ je puise. moi, nuit \\ d'où \\ issu, je \\ puise ${ }^{31}$.
}

\title{
3. Poèmes s'ouvrant
}

L'arbre, thème central de la troisième section intitulée «s'ouvrant», mieux encore que le corps humain, figure ce lieu de passage, cet entre-deux. Et la section ellemême est un entre-deux, un pivot entre la première moitié du livre - où dominent les images chthoniennes, souterraines, rouges et noires - et la seconde - plus lumineuse, celle de l'éclat, comme l'indiquent les titres: entre le feu et le soleil; l'oubli, l'éclat. Passage, ouverture - voire «fracture» - où s'insinue la lumière, couloir entre la terre et l'air, entre le noir et l'éclat, stigmatisé dans l'arbre, anthropomorphe (mains I de l'arbre dans l'air ${ }^{32}$ ), figure en miroir «qui déploie la terre en ciel» ${ }^{33}$. Du rouge au bleu

(27) F. Ducros, Surgies syllabes arrachées, p. 16.

(28) ID, Jointure dans Poésie, figures traversées, p.138.

(29) F. Ducros, Surgies syllabes arrachées, p. 33.
(30) F. Ducros, Poésie, figures traverséss, p. 127.

(31) F. Ducros, Surgies syllabes arrachées, p. 55.

(32) Ibid., p. 39.

(33) Ibid., p. 30. 
et au blanc, du feu, au soleil, le livre s'articule comme un lancé et un rebondissement d'images, de couleurs et formes récurrentes, mais en devenir, nous surprenant. On perçoit alors de mieux en mieux, au fil des poèmes, le rapport de cette poésie à l'art plastique ${ }^{34}$, rapport fondamental qui met en cause le fait de voir et de tracer. La peinture en particulier, tient une place d'exception dans la deuxième partie du livre. Elle accompagne le poète, tantôt l'inspirant (poèmes sur les taureaux de Jean Azemard, aquarelles), tantôt puisant son inspiration dans ses poèmes (Delphes a fait l'objet d'un livre manuscrit et peint, en collaboration avec Anne Slacik).

La dédicace de la suite intitulée «rouge», à Su Dongpo ${ }^{35}$, grand peintre et poète taoïste, nous conduit sur cette voie. C'est à lui que Franc Ducros emprunte la description de l'acte créateur, contenue dans la lettre À propos des «Bambous penchés dans la vallée de Yundang», peints par Wen Yuke(1079):

Quand les bambous commencent à pousser, ce sont des bourgeons d'un pouce; mais les nœuds et les feuilles y sont déjà inclus. Ce qui se passe entre le moment où ils ressemblent à des ventres de cigale ou de serpent et celui où ils deviennent comme des épées dégainées de quatre-vingt pieds est leur croissance. À présent, les peintres en reproduisent tous les nœuds, accumulent toutes leur feuilles; mais alors, comment y aurait-il encore des bambous! C'est pourquoi pour peindre des bambous, il faut d'abord les former dans sa tête, bien les considérer de son regard intérieur en tenant le pinceau, puis, quand on voit la forme qu'on veut peindre, vite s'y conformer, manier son pinceau jusqu'à ce qu'on l'ait atteinte et poursuivre ce qu' on voit comme le faucon s'abat sur le lièvre qui détale; si on se relâche un tant soit peu, cette forme disparaît ${ }^{36}$.

Pour Franc Ducros, la tâche du poète est la même que celle du peintre, celle de, par son geste, «donner naissance à un bambou»: «L'énergie que le geste expulse et trace sur la page est celle-là même - universelle - qui donne naissance au végétal et en assure la croissance selon le devenir inscrit dans sa structure virtuelle» ${ }^{37}$. On retrouve dans sa poésie la quête de la forme potentielle, à sa naissance, possédant déjà in fieri toutes les possibilités. Et si la forme peut être tantôt la ligne verticale de l'arbre, ou l'oblique de la fracture zigzaguante, c'est aussi celle du point ${ }^{38}$, départ et aboutissement de toutes les formes, vie universelle à l'état virtuel:

où sourd de terre toujours noire, même

verte la future tige - ce point, que

l'œil aura fixé jusqu'à l'aveuglement" .

C'est pourquoi la poésie se dépouille du superflu, à savoir le récit, - comme déjà la poésie classique chinoise dont on ressent dans «rouge» des échos en guise d'hommage - même dans les rares cas où elle amorce à peine une «histoire»:

(34) On ne peut ignorer l'importance de l'art dans l'œuvre critique de Franc Ducros; ses séminaires sur «le poétique» mais aussi sur l'esthétique, ses écrits sur Titien, Michel-Ange, Bernin, Giacometti, De Staël etc. Pour lui, comme pour toute la poésie contemporaine, cette relation est fondamentale. La peinture, comme la poésie, trace ce qui est lancé en avant, à venir.

(35) Su Shi (1036-1101), écrivain, peintre et calligraphe chinois, connu sous plusieurs pseudonymes dont Su Tong Po et Su Dongpo.
(36) Su DongPO, À propos des «Bambous penchés dans la vallée de Yundang», peints par We Yuke, dans Sur moi-même, Arles, Picquier, 2003, p. 124.

(37) F. Ducros, Le Poétique et le réel, p. 121.

(38) «[...] sur ce 'point' toute chose, donc aussi la parole, pour la première fois 'commence'». Commentaire à «ce point final du ciel qu'est la lune» de Reverdydans F. Ducros, Poésie, figures traversées, p. 86.

(39) F. Ducros, Surgies syllabes arrachées, p. 67. 
gouverneur
de villes et d'arbres
d'hommes, de fleurs

qui ne viendra

attendant

C'est également la raison pour laquelle les incipit possèdent autant de force et contiennent les éléments qui iront s'articuler, de façon inattendue, dans la suite. Articulation poétique qui est l'une des caractéristiques de cette poésie et qui mérite que l'on s'y arrête un instant, en essayant de l'illustrer par quelques passages choisis de «rouge».

On pourrait définir ce procédé comme une déclinaison de figures rebondissantes, à savoir l'articulation thématique entre deux ou plusieurs «figures», par exemple ici la feuille et la neige, dont on peut suivre le chemin croisé et contrasté (par la couleur, la forme, mais aussi la sensation tactile de chaud et froid), dans chaque partie d'une section, jusqu'à d'autres sections, voire au livre entier. Si une telle jointure ${ }^{41}$ est poétique, c'est qu'elle est à la fois impensable, «inouïe», et encore chargée de la part de mystère qu'elle ne dévoile pas entièrement, même montrée, même visible. Ainsi, au fil des premiers mouvements qui composent le poème, sont finement tracées les lignes de liaison entre quelques éléments:

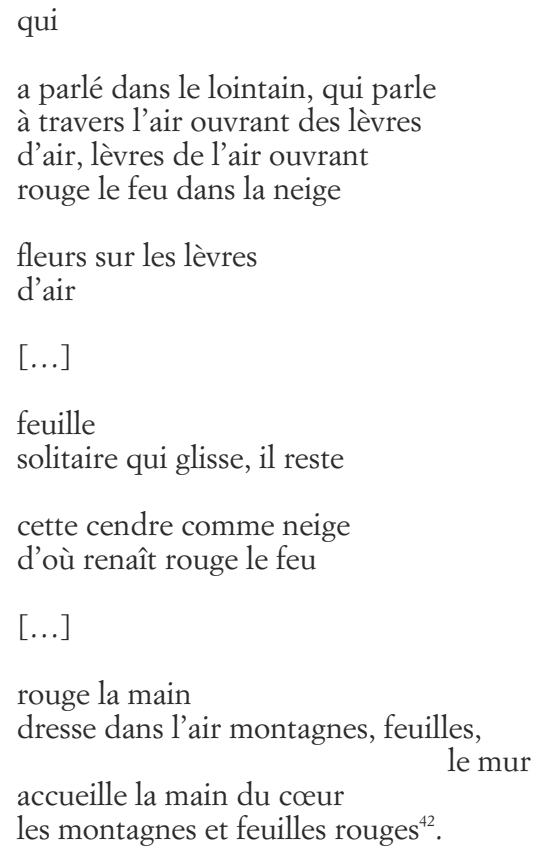


Dans cette déclinaison qui passe par: parole-fleur, lèvre et air, rouge et blanc, main et nuées, eau et chair, mur et cœur, les rapports d'équivalence entre certaines figures, comme feuille-main (ainsi, par exemple, «main de l'arbre» est figure naissant de l'antropomorphisme de l'arbre mais aussi de la forme et la couleur de cette feuille) finissent par rebondir dans d'autres rapports moins évidents et pourtant suggérés à notre écoute. Ainsi, c'est parce qu'il y a eu d'abord les associations feuille-rouge, main-feuille, rebondissant dans «main du cœur», que peut surgir:

parole comme

[...]

l'unique

feuille

le rouge des lointains

dans la poitrine ${ }^{43}$.

$* * *$

L'œuvre poétique de Franc Ducros, tout comme son œuvre critique, a posé, d'entrée, la question de la figure, du rapport entre expérience - voir, avant tout - et parole. Si l'expérience poétique se présente comme une catabase, une descente et ascension vertigineuse entre deux abîmes, elle se donne en tant que figure. En fuite et en abîme constant entre le réel et la langue, la figure reste cette expérience qui, comme chez Du Bouchet, «parvenue à son terme inquiet» ${ }^{44}$, est déjà en train de mourir:

le retour

$[\ldots] \ll$ dans

vertigineux l'image meurt

instantanée» ${ }^{45}$.

Voir est ce qui nous est donné, un instant, ouverture et épanouissement:

comme s'ouvrent les yeux aux lointains

déserté le miroir

d'où se sont élancés

en sept jours devenus

ceux-là qui s'épanouissent - les yeux

dans les lointains

enfouis ${ }^{46}$.

(43) Ibid., p. 43.

(44) «Où est-elle alors? autant dire, nulle part. À l'extrémité, elle commence. Instant singulier dont la mémoire nous est presque aussitôt retirée, mais dont nous avons aussi le pressentiment qu'il peut ne pas être unique» A. Du Bouchet, Image parvenue à son terme inquiet, Fata Morgana, 1984.
(45) F. Ducros, Ibid., p. 103. Il s'agit d'une traduction-adaptation d'une phrase des Taccuini de Dino Campana: «Nell'eterno ritorno vertiginoso l'immagine muore immediatamente». Dino CAMPANA, Taccuinetto Faentino, Firenze, Vallecchi, 1960. (46) Ibid., p. 111. 
Mais ce que les yeux ont pu percevoir, dans cet acte profondément intense et primordial qu'est s'ouvrir et s'abîmer, s'enfouir, - éclat ou tumulte - se perd dans les mots. L'instant poétique est cet éclair - d'où des poèmes brefs, instantanés - qui tient en équilibre dans une figure qui, disparaissant apparaissant, reste unique. La poésie nous offre alors, et nous lui en sommes reconnaissants, la possibilité d'un accès à l'expérience unique se donnant en figure - «ce qui n'a pas bougé» - par une parole toujours future:

\author{
paroles pour \\ plus tard, d'où \\ attendre, inaudibles \\ - ce qui \\ n'a pas bougé: tumulte \\ le vertige \\ silence \\ en fuite. ${ }^{47}$
}

\title{
Note bio-bibliographique
}

Franc Ducros est né en 1936 à Montpellier et il a enseigné la littérature italienne à l'université de Montpellier. Il a fondé une revue, «Prévue», et animé un seminaire de recherche sur «le poétique». Il a publié des essais sur la poésie (en particulier sur Dante, Michel-Ange, Hölderlin, Mallarmé, Reverdy, Du Bouchet): Tommaso Campanella, poète, Paris, PUF, 1969; Le poétique et le réel, Paris, Les Méridiens-Klincksieck, 1987; Poésie, figures traversées, Saint-Maximin, Théétète, 1995; L'odeur de la panthère: Dante, la poésie, Saint-Maximin, Théétète, 1997; Pour Mallarmé, SaintMaximin,Théétète, 1998; Notes sur la poésie, Nîmes, Champ social, 2006; Lectures Poétiques, Nîmes, Champ social, 2006. Il a traduit: «Ombre lointaine». 46 fragments de Léonard de Vinci, Alinéa, 1983; Pascal Gabellone, L'inhabité, «Main d'œuvre», «Prévue», 1993; Poèmes de Michel-Ange, Saint-Maximin, Théétète, 1996; Gabriel Magaña, Le rien rugueux, Saint-Maximin, Théétète, 1998; «Comme on cherche un trésor», poèmes d'Umberto Saba, Genève, La Dogana, 2004. Ses livres de poésie parus depuis 1993 - Les yeux, la terre, «Main d'œuvre», «Prévue», 1993; s'ouvrant l'arbre, Saint-Maximin, Théétète, 1997; du noir cela, Saint-Maximin, Théétète, 2000; entre le feu et le soleil, livre manuscrit peint, en collaboration avec Anne Slacik, Montpellier, 1998; Delphes, id., 1999 - ont été repris et augmentés dans son dernier livre, surgies, syllabes, arrachées, Nîmes, Théétète, 2003. Ses poèmes ont été partiellement traduits en espagnol, roumain, sicilien, italien (dans «L'Immaginazione», gennaio-febbraio 2005 et dans «Anterem», n. 72 et n. 73, 2006).

MARGHERITA ORSINO

(47) Ibid., p. 133. Ce poème, l'avant-dernier du livre, est inclus dans la section «neige du 21 avril» dédiée à André Du Bouchet dont l'enterrement a eu lieu à cette date, à Truinas. L'italique souligne la valeur «programmatique» de cette déclaration sur la poésie d'André Du Bouchet et sur la poésie tout court, lorsqu'elle atteint une valeur universelle. «[...]cela| n'a pas bougé», ce sont les tout derniers mots du dernier livre d'André Du Bouchet, Tumulte, Fata Morgana, 2001. 